\title{
Three-Dimensional Evaluation of Change in Maxillary Alveolar Arch after Expansion in Unilateral Cleft Lip and Palate Patients
}

\author{
Issei Takahashi $^{1)}$, Teruo Sakamoto ${ }^{1)}$, Takenobu Ishiii ${ }^{2)}$ and Kenji Sueishii ${ }^{2)}$ \\ ${ }^{1)}$ Department of Orthodontics, Tokyo Dental College, \\ 1-2-2 Masago, Mihama-ku, Chiba 261-8502, Japan \\ 2) Department of Orthodontics, Tokyo Dental College, \\ 2-9-18 Kanda-Misakicho, Chiyoda-ku, Tokyo 101-0061, Japan
}

Received 7 March, 2019/Accepted for Publication 30 August, 2019

Published Online in J-STAGE 10 June, 2020

\begin{abstract}
Narrowing of the maxillary dental arch is a major cause of occlusal abnormalities in cleft lip and palate patients. Although the dental arch may be expanded in such cases, relapse will often occur during the subsequent retention period. In this study, the stability of expansion of the maxillary arch was investigated by examining 3-dimensional change in the maxillary arch during the treatment and post-retention periods. Three-dimensional measurements was performed on maxillary plaster models obtained from 8 unilateral cleft lip and palate patients (mean age, 12.5 years) who had undergone maxillary arch expansion using an edgewise appliance and quad helix (CLP group). The controls consisted of 8 unilateral cleft lip and alveolus patients (mean age, 12.9 years). Measurements were made during the pretreatment, post-treatment, and post-retention periods. In the CLP group, horizontal relapse was observed in the alveolar and dental arches between the second premolars, together with vertical relapse on the cleft side of the central incisor, lateral incisor, and canine. The sites where relapse occurred demonstrated decreased growth before orthodontic treatment. A correlation was observed between the extents of expansion and relapse. These findings suggest that excessive horizontal or vertical tooth movement in areas showing developmental failure should be avoided in order to increase stability after orthodontic treatment.
\end{abstract}

Key words: Cleft lip and palate - Maxillary plaster models 3-dimensional change - Maxillary dental arch

This paper was originally a thesis submitted to the Graduate School of Tokyo Dental College on February 9, 2018, by Issei Takahashi. 


\section{Introduction}

Repair of cleft lip and palatoplasty are considered to be the most important factors involved in disturbance of maxillary growth and development due to surgical trauma and scar tissue formation in patients with unilateral cleft lip and palate ${ }^{7,18)}$. Dental crowding is characteristically severe in such patients, and anterior or posterior crossbite due to skeletal sagittal deficiency or a shortage of space in the dental arch are often observed ${ }^{21)}$. Orthodontic treatment often results in transverse expansion of the dental arches owing to a decrease in maxillary growth ${ }^{12,13}$. The results of treatment are not always stable, however, and relapse is often noted ${ }^{1,17,19,25)}$. Although several studies have investigated maxillary arch expansion during orthodontic treatment, few have explored subsequent change during the retention period. Furthermore, these reports have only analyzed changes in horizontal parameters such as alveolar arch width. Therefore, the effect of orthodontic treatment on vertical parameters remains inconclusive.

The aim of this study was to evaluate the stability of orthodontic treatment by using a three-dimensional (3D) digitizer to measure both horizontal and vertical change.

\section{Materials and Methods}

The protocol of this study was reviewed and approved by the Institutional Review Board of Tokyo Dental College (approval number: $745)$.

\section{Materials and methods}

Patients with unilateral cleft lip and palate who presented at the Orthodontic Department of the Tokyo Dental College Chiba Hospital and who met the following conditions were included in the study: use of quad helix as an expansion device; dental age IIIB or greater during initial examination; no missing maxillary central incisors or first molars; history of non-extraction dental treatment using an edgewise appliance; an expansion period of $\leq 18$ months; use of retainers as instructed and attendance at regular examinations; and availability of all materials used, from initial examination to at least 24 months after retention. Syndromic patients and those with incomplete clefts were excluded from the study.

The test (CLP) group consisted of maxillary dental casts obtained from 8 unilateral cleft lip and palate patients ( 5 boys and 3 girls) who met the above requirements. To investigate differences in stability due to cleft palate and palatoplasty and determine how cleft palate and palatoplasty had affected the cleft region, a control (CLA) group consisting of casts from 8 unilateral cleft lip and alveolus patients ( 4 boys and 4 girls) of similar age was included. Maxillary dental casts of these patients obtained at before commencement of orthodontic treatment (T1), at after orthodontic treatment (debonding; T2), and at least 24 months after retention (T3) were used.

In the CLP group, the T1 age was $12.5 \pm 0.8$ years, the T2 age $15.2 \pm 0.7$ years, and the T3 age $17.5 \pm 0.5$ years. In the CLA group, the T1 age was $12.9 \pm 1.0$ years, the T2 age $15.2 \pm 1.2$ years, and the T3 age $17.4 \pm 1.2$ years.

Cleft lip repair was performed at approximately 6 months using the Millard technique, and palate closure at approximately 18 months using the Wardill technique at Chiba Children's Hospital. Patients in the CLA group also underwent surgery involving repair of cleft lip utilizing the Millard technique at approximately 6 months of age. All patients underwent secondary alveolar bone grafting with particulate cancellous bone and bone marrow obtained from the anterior iliac crest before eruption of the canines.

The maxillary arch was expanded laterally with a quad helix appliance (Fig. 1-a) in all the patients. The period for lateral expansion was 12-18 months. After orthodontic treatment, a circumferential retainer was used to retain the expansion (Fig. 1-b). During the first 12 months after removal of the edgewise appliance, a retainer was used at all times, 

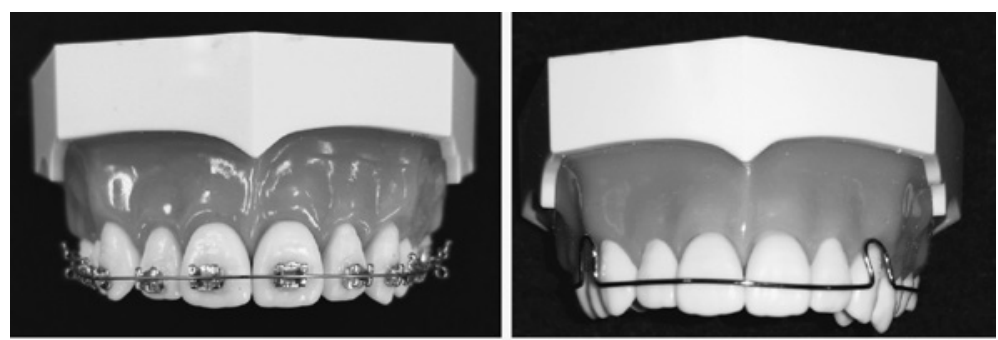

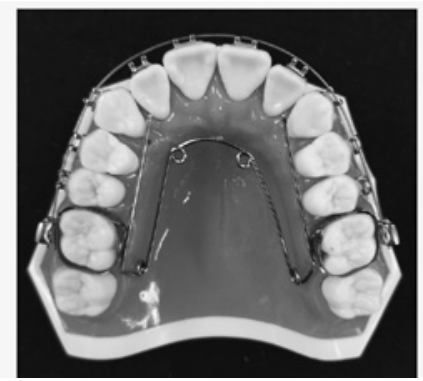

(a)

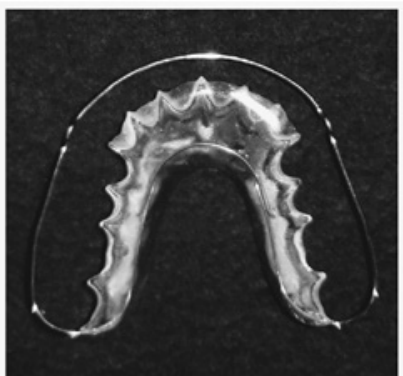

(b)

Fig. 1 a: Edgewise appliance with quad helix appliance. b: Circumferential retainer.

except when eating or brushing the teeth, while for the ensuing 12 months it was only used while the patient was sleeping. The usage status of the circumferential retainer was confirmed at 1 month after fitting. Subsequently, the patients attended examinations every 3 months so that their appliance could be adjusted and status of oral hygiene confirmed.

\section{Morphological analysis using 3D measurement system}

1) Measurement process

A 3D measurement system was used to measure the dental casts. This process involves a digitizer scan, surface model fabrication, and data measurement. The maxillary dental casts were imaged with a 3D scanner (3ShapeR700 Orthodontic Scanner, Great Lakes Orthodontics, USA) and 3D images reconstructed. A 3D point group processing software (Imageware) was used for measurement.

The maxillary dental model was measured by one researcher. One month later, localization and measurement of landmarks were

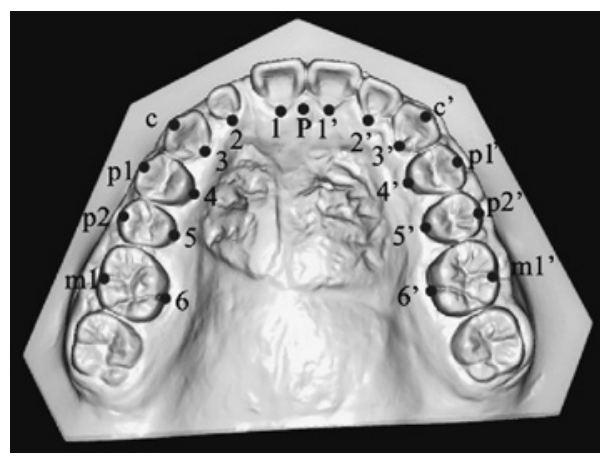

Fig. 2 Measurement points.

repeated by the same researchers under the same conditions. One more month later, this was repeated. The average value of the 3 measurements was used.

2) Measurement points and reference plane

(1) Measurement points (Fig. 2)

1. P: widest point of the maxillary incisive papilla.

2. 1: lowest point of the palatal side of the cervix of the central incisor on the cleft 


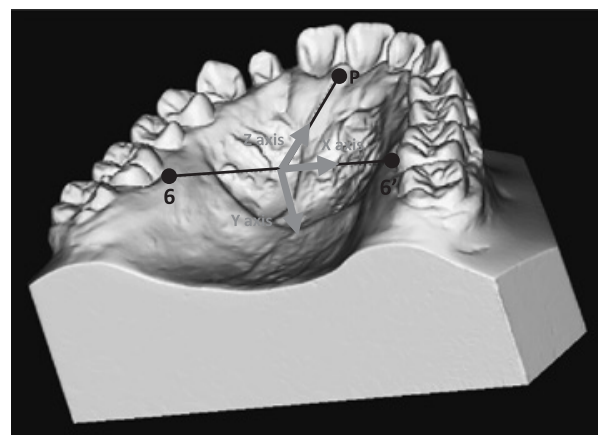

Fig. 3 Reference plane.

Location of 3 points for creation of reference plane. $\mathrm{P}$, widest point on incisive papilla; 6 , lowest point on palatal side of cervix of first molar on cleft side; 6', lowest point on palatal side of cervix of first molar on noncleft side.

side.

3. 1': lowest point on the palatal side of the cervix of the central incisor on the noncleft side.

4. 2: lowest point on the palatal side of the cervix of the lateral incisor on the cleft side.

5. 2': lowest point of the palatal side of the cervix of the lateral incisor on the noncleft side.

6. 3: lowest point on the palatal side of the cervix of the canine on the cleft side.

7. 3': lowest point on the palatal side of the cervix of the canine on the non-cleft side.

8. 4: lowest point on the palatal side of the cervix of the first premolar on the cleft side.

9. 4': lowest point on the palatal side of the cervix of the first premolar on the noncleft side.

10. 5: lowest point on the palatal side of the cervix of the second premolar on the cleft side.

11. 5': lowest point on the palatal side of the cervix of the second premolar on the non-cleft side.

12. 6: lowest point on the palatal side of the cervix of the first molar on the cleft side.

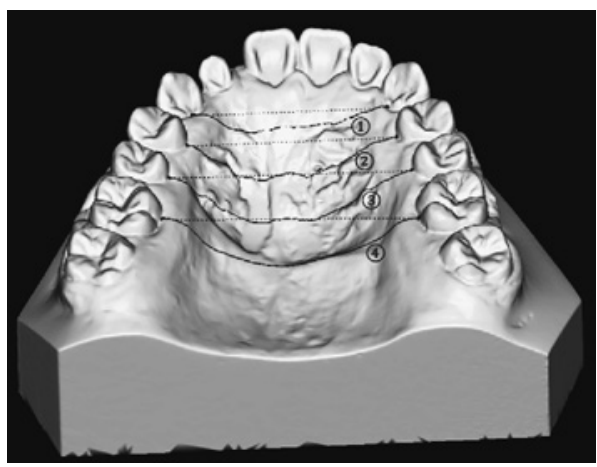

Fig. 4 Palatal projecting lines were created on palatal plane by using straight line to join 2 measurement point pairs in direction of Y-axis.

13. 6': lowest point on the palatal side of the cervix of the first molar on the non-cleft side.

14. c: cusp of the canine on the cleft side.

15. c': cusp of the canine on the non-cleft side.

16. p1: cusp tip on the buccal side of the first premolar on the cleft side.

17. p1': cusp tip on the buccal side of the first premolar on the non-cleft side.

18. p2: cusp tip on the buccal side of the second premolar on the cleft side.

19. p2': cusp tip on the buccal side of the second premolar on the non-cleft side.

20. m: cusp tip on the mesio-buccal side of the first molar on the cleft side.

21. m': cusp tip on the mesio-buccal side of the first molar on the non-cleft side.

(2) Reference plane (Fig. 3)

The reference plane comprised a line connecting the lowest points on the palatal sides of the cervices of the left and right first molars and the widest point on the papilla of the maxillary incisor. Next, the point bisecting the lowest points on the palatal sides of the cervices of the left and right first molars was used as a starting point. The axis drawn in the direction of the lowest point on the palatal side of the cervix of the left first molar was the $\mathrm{X}$-axis, while the axis drawn in the direction of the widest point on the papilla of the maxillary incisor was the Z-axis. The axis deter- 


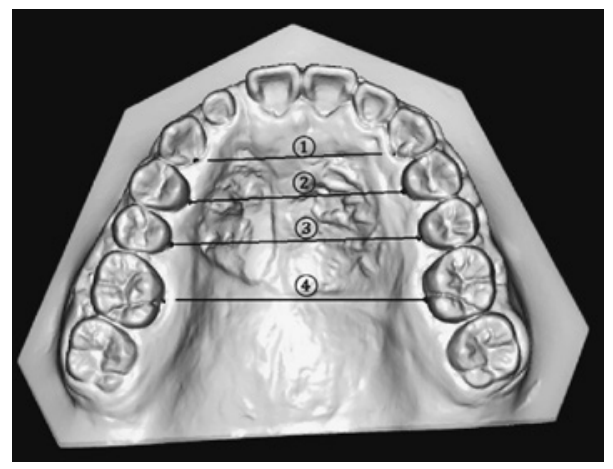

Fig. 5 Alveolar arch width was measured as distance between cervical points.

mined by these two axes was the Y-axis (palate direction was positive).

(3) Palatal imaging lines (Fig. 4)

A curved line was created on the palatal plane, and a straight line joining the two points for measurement was projected in the direction of the Y-axis to create the palatal projection line.

1. Map 3-3': curved line when 3-3' was imaged on the palatal plane perpendicular to the palate.

2. Map 4-4': curved line when 4-4' was imaged on the palatal plane perpendicular to the palate.

3. Map 5-5': curved line when 5-5' was imaged on the palatal plane perpendicular to the palate.

4. Map 6-6': curved line when 6-6' was imaged on the palatal plane perpendicular to the palate.

3) Measurement items

(1) Alveolar arch width (Fig. 5)

1. Alveolar arch width between canines (3-3' width).

Distance between the lowest points on the palatal sides of the cervical lines of the left and right canines.

2. Alveolar arch width between first premolars (4-4' width).

Distance between lowest points on the palatal sides of the cervical lines of the left and right first premolars.

3. Alveolar arch width between second pre-

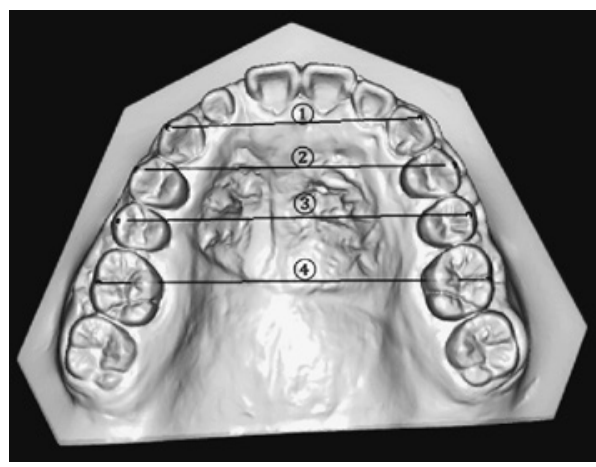

Fig. 6 Dental arch width was measured as distance between cusps.

molars (5-5' width).

Distance between lowest points on the palatal sides of the cervical lines of the left and right second premolars.

4. Alveolar arch width between first molars (6-6' width).

Distance between lowest points on the palatal sides of the cervical lines of the left and right first molars.

(2) Dental arch width (distance between cusp tips) (Fig. 6)

1. Width between canine cusps (c-c' width). Distance between left and right canine cusps.

2. Width between first premolar buccal side cusp tips (p1-p1' width).

Distance between the cusp tips on the buccal sides of the left and right first premolars.

3 . Width between second premolar buccal side cusp tips (p2-p2' width).

Distance between the cusp tips on the buccal sides of the left and right second premolars.

4. Width between first molar mesial buccal side cusp tips (m-m' width).

Distance between the cusp tips on the buccal sides of the left and right first molars.

(3) Palate length (length) (Fig. 7)

The length of the palate was measured from the incisive papilla to the lowest point of the cervix on the palatal side of the first 


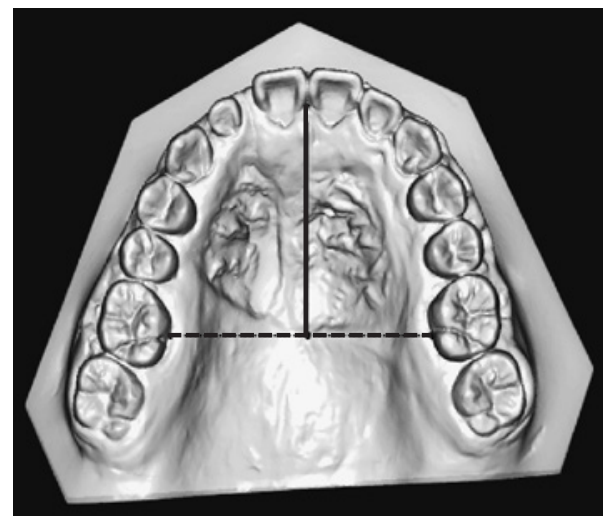

Fig. 7 Length of palate was measured from incisive papilla to lowest point on palatal side of cervix of first molars in horizontal plane.

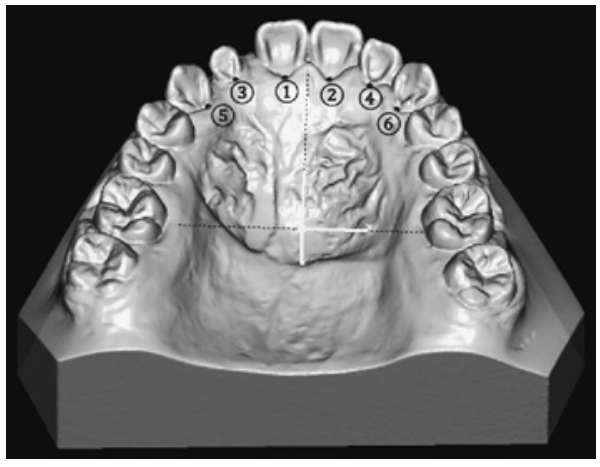

Fig. 8 Height of anterior alveolar area. Lowest point on palatal side of cervix of each tooth.

molars in the horizontal plane.

(4) Heights of alveolar area and palate

(i) For the central and lateral incisors, and canines, the distance from the starting point was measured 3-dimensionally using the Y coordinates (Figs. 8, 9).

1. Lowest point on the palatal side of the cervix of the central incisor on the cleft side (1 height).

2. Lowest point on the palatal side of the cervix of the central incisor on the noncleft side ( 1 ' height).

3. Lowest point on the palatal side of the cervix of the lateral incisor on the cleft

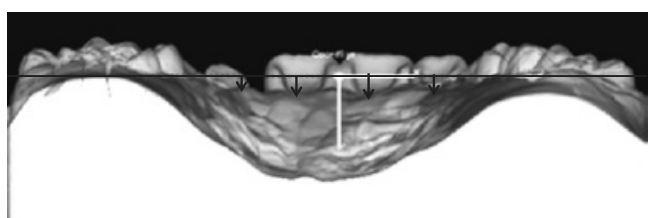

Fig. 9 Height of anterior alveolar area showing value of $\mathrm{Y}$ coordinate at each measurement point.

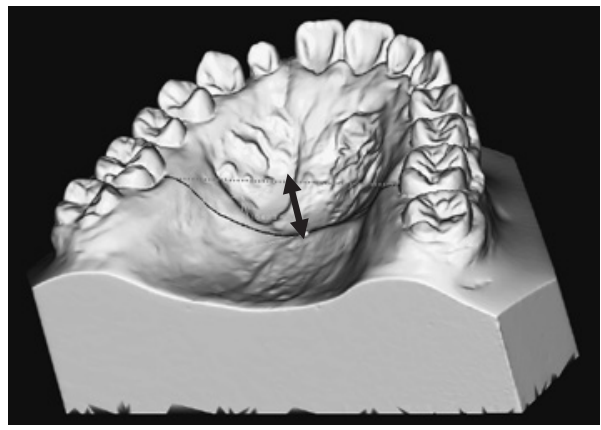

Fig. 10 Palatal height was measured from line passing from lowest point on palatal side of cervix of first molars to deepest point on palate perpendicular to reference plane.

side (2 height).

4. Lowest point on the palatal side of the cervix of the lateral incisor on the noncleft side (2' height).

5 . Lowest point on the palatal side of the cervix of the canine on the cleft side (3 height).

6. Lowest point on the palatal side of the cervix of the canine on the non-cleft side (3' height).

(ii) For canines, premolars, and molars, the palatal heights were measured from a line passing through the lowest point on the palatal side of the cervix of the first molars on the cleft side to the deepest point on the palate perpendicular to reference plane (Fig. 10).

1. Canine maximum height (3-3' height). Maximum distance between 3-3' and Map 3-3'.

2. First premolar maximum height (4-4' 


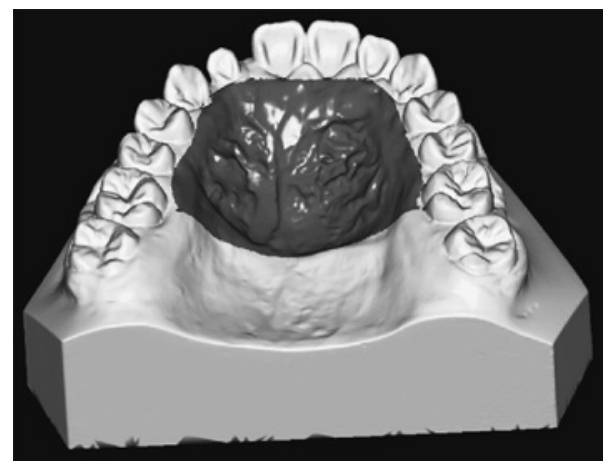

Fig. 11 Palate surface area enclosed by Map 6-6' and cervical lines on palatal sides of anterior teeth to first molar.

height).

Maximum distance between 4-4' and Map 4-4'.

3. Second premolar maximum height (5-5' height).

Maximum distance between 5-5' and Map 5-5'.

4. First molar maximum height (6-6' height).

Maximum distance between 6-6' and Map 6-6'.

(5) Palatal surface area (Map; Fig. 11)

Palatal surface area enclosed by Map 6-6 and palatal lateral teeth cervix lines in the teeth anterior to the first molars.

(6) Palatal cross-sectional area (Fig. 12)

The area of the planes enclosed by the straight and curved lines described below were calculated.

1. Canine cross-sectional area ( $3 \mathrm{sps}$ ).

Area of plane enclosed by 3-3' and Map3-3'.

2. First premolar cross-sectional area (4 sps).

Area of plane enclosed by 4-4' and Map 4-4'.

3. Second premolar cross-sectional area (5 sps).

Area of plane enclosed by 5-5' and Map5-5'.

4. First molar cross-sectional area (6 sps). Area of plane enclosed by $6-6$ ' and Map

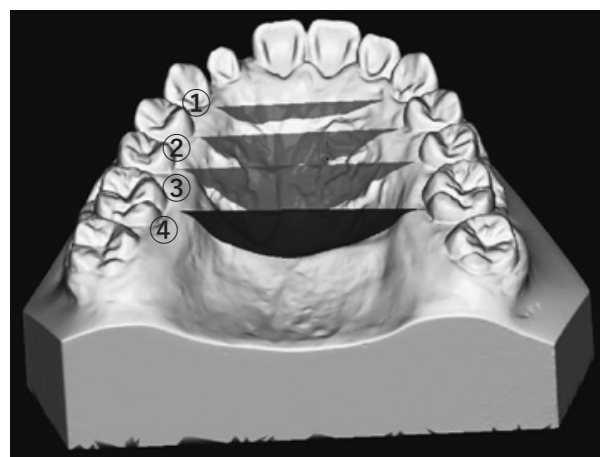

Fig. 12 Palatal cross-sectional area.

Area of planes enclosed by straight and curved lines were calculated.

6-6'.

\section{Statistical analysis}

The Friedman's test was used to analyze mean differences in measurements at T1, T2, and T3. When a statistically significant difference was found, pairwise comparisons were performed using the Wilcoxon signed rank test.

The Mann-Whitney $U$ test was used for comparisons between the CLP and CLA groups. The level of significance was set at $<0.05$. Spearman's correlation coefficient was used to investigate the relationship between treatment and post-treatment changes. Correlation coefficients $>0.7$ indicated strong correlations.

The software used for the statistical analyses was SPSS Statistics for Windows, version 24.0 (IBM Corp, USA).

\section{Results}

\section{Alveolar arch width}

1) Intragroup comparison (Tables 1 and 2)

In the CLP group, significant increases in the widths of 3-3', 4-4, 5-5', and 6-6', and a significant decrease in the $5-5$ ' width were noted during the treatment period. Similarly, a significant increase in 3-3' width was 


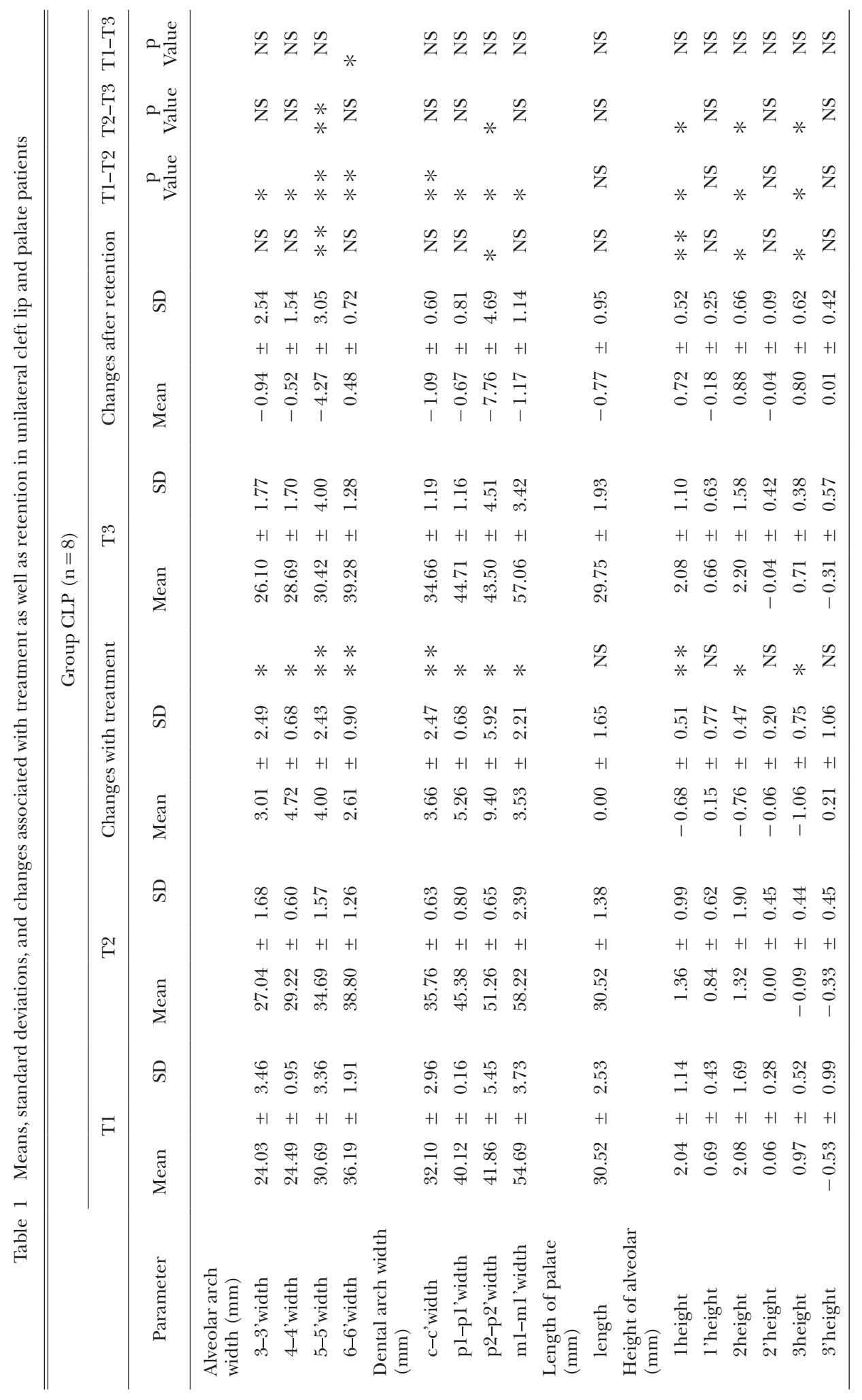


3D Model of Maxillary Arch Expansion

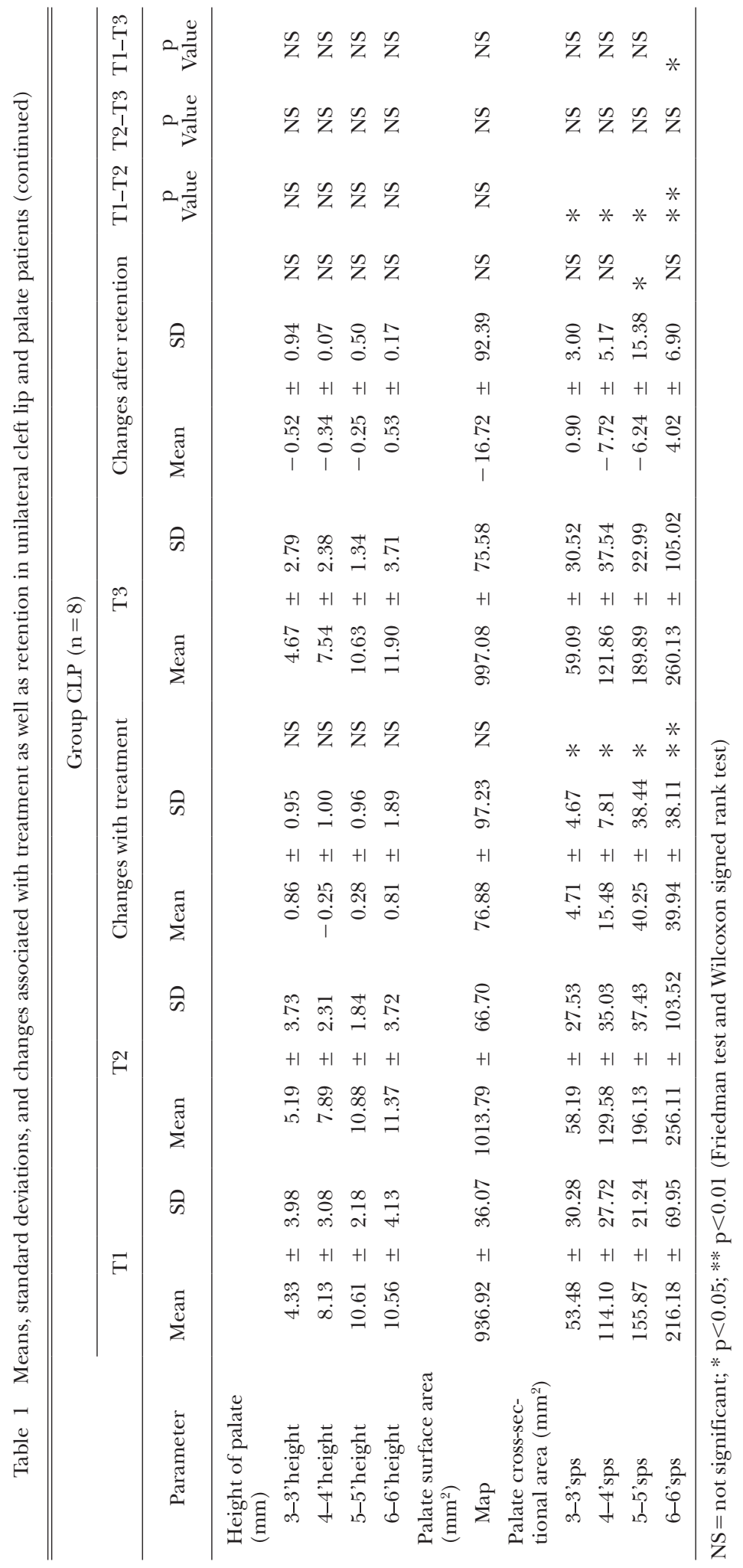




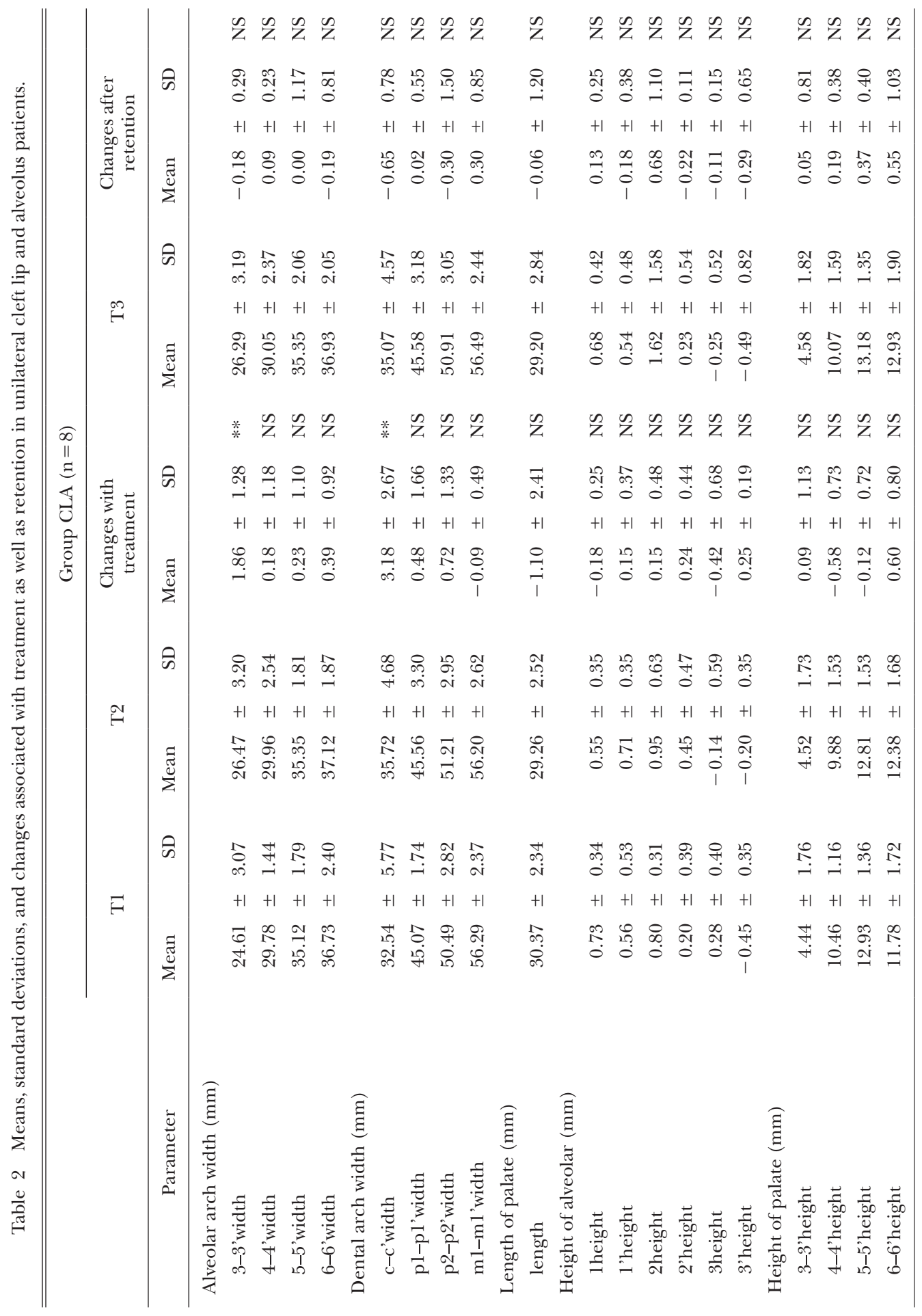


observed in the CLA group during the treatment period.

2) Intergroup comparison (Tables 3 and 4)

At T1, the 4-4' and 5-5' widths were significantly smaller in the CLP group, while at T3, the 5-5' width was significantly smaller than those in the CLA group. The extent of change during treatment in the CLP group was significantly greater than that in the CLA group at sites 5-5' and 6-6'. Likewise, the degree of post-treatment change was significantly higher in the CLP group than in the CLA group at the 5-5' site.

\section{Dental arch width}

1) Intragroup comparison (Tables 1 and 2)

The widths of c-c', p1-p1', p2-p2', and m1$\mathrm{ml}$ ' were showed a significant increase in the CLP group during the treatment period, whereas the width of p2-p2' showed a significant decrease post-treatment. In the CLA group, a significant increase was noted in the width of c-c' during the treatment period.

2) Intergroup comparison (Tables 3 and 4)

At T1, the widths of p1-p1' and p2-p2' were significantly smaller in the CLP group than in the CLA group, whereas at T3, the width of p2-p2' was significantly smaller. Change during treatment was significantly more pronounced in the CLP group than in the CLA group at sites p2-p2' and m1-m1'. Similarly, significantly larger amounts of posttreatment change were noted in the CLP group at the p2-p2' site.

\section{Palatal length}

1) Intragroup comparison (Tables 1 and 2)

No significant differences were noted at any of the time points in the CLP or CLA groups.

2) Intergroup comparison (Tables 3 and 4)

No significant differences were noted at T1, T2, or T3. Likewise, no significant differences were observed in the extent of change between at during and post-treatment.

\section{Heights of alveolar area and palate}

1) Anterior alveolar height

(1) Intragroup comparison (Tables 1 and 
Table 3 Comparison of means between groups.

\begin{tabular}{|c|c|c|c|}
\hline Parameter & $\mathrm{T} 1$ & $\mathrm{~T} 2$ & T3 \\
\hline \multicolumn{4}{|c|}{ Alveolar arch width (mm) } \\
\hline 3-3'width & NS & NS & NS \\
\hline 4-4'width & NS & NS & NS \\
\hline 5-5'width & CLP $<$ CLA * & NS & CLP $<$ CLA * \\
\hline 6-6'width & NS & NS & NS \\
\hline \multicolumn{4}{|c|}{ Dental arch width (mm) } \\
\hline c-c'width & NS & NS & NS \\
\hline p1-pl'width & NS & NS & NS \\
\hline p2-p2'width & CLP $<$ CLA * & NS & CLP $<$ CLA * \\
\hline m1-m1'width & NS & NS & NS \\
\hline \multicolumn{4}{|l|}{ Length of palate $(\mathrm{mm})$} \\
\hline length & NS & NS & NS \\
\hline \multicolumn{4}{|l|}{ Height of alveolar (mm) } \\
\hline 1height & CLA $<$ CLP $*$ & NS & NS \\
\hline l'height & NS & NS & NS \\
\hline 2height & $\mathrm{CLA}<\mathrm{CLP} *$ & NS & NS \\
\hline 2'height & NS & NS & NS \\
\hline 3height & CLA $<$ CLP $*$ & NS & NS \\
\hline 3'height & NS & NS & NS \\
\hline \multicolumn{4}{|l|}{ Height of palate $(\mathrm{mm})$} \\
\hline 3-3'height & NS & NS & NS \\
\hline 4-4'height & NS & NS & NS \\
\hline 5-5'height & CLP $<$ CLA * & CLP $<$ CLA * & CLP $<$ CLA ** \\
\hline $6-6$ 'height & NS & NS & NS \\
\hline \multicolumn{4}{|c|}{ Palate surface area $\left(\mathrm{mm}^{3}\right)$} \\
\hline Map & NS & NS & NS \\
\hline \multicolumn{4}{|c|}{ Palate cross-sectional area $\left(\mathrm{mm}^{3}\right)$} \\
\hline 3-3'sps & NS & NS & NS \\
\hline 4-4'sps & CLP $<$ CLA * & NS & NS \\
\hline $5-5$ ’sps & CLP $<$ CLA $* *$ & CLP $<$ CLA $* *$ & CLP $<$ CLA ** \\
\hline $6-6$ 'sps & CLP $<$ CLA $* *$ & CLP $<$ CLA * & CLP $<$ CLA * \\
\hline
\end{tabular}

$\mathrm{NS}=$ not significant; $* \mathrm{p}<0.05 ; * * \mathrm{p}<0.01$ (Mann-Whitney $U$ test)

2)

During the treatment period, the heights of 1,2 , and 3 showed a significant reduction in the CLP group, whereas during post-treatment period they showed a significant increase. No significant differences were observed at T1, T2, or T3 in the CLA group. (2) Intergroup comparison (Tables 3 and 4) cantly greater in the CLP group than those in the CLA group. The extent of change during the treatment period was significantly greater in the CLP group than in the CLA group at sites 1, 2, and 3. Likewise, the extent of posttreatment change was significantly larger in the CLP group than in the CLA group at site 3.

2) Height of palate 
Table 4 Comparison of changes associated with treatment and retention.

\begin{tabular}{|c|c|c|}
\hline Parameter & $\mathrm{T} 1-\mathrm{T} 2$ & T2-T3 \\
\hline \multicolumn{3}{|c|}{ Alveolar arch width (mm) } \\
\hline 3-3'width & NS & NS \\
\hline 4-4'width & NS & NS \\
\hline 5-5'width & $\mathrm{CLA}<\mathrm{CLP} * *$ & CLA $<$ CLP $* *$ \\
\hline 6-6'width & $\mathrm{CLA}<\mathrm{CLP} * *$ & NS \\
\hline \multicolumn{3}{|l|}{ Dental arch width $(\mathrm{mm})$} \\
\hline c-c'width & NS & NS \\
\hline p1-p1'width & NS & NS \\
\hline p2-p2'width & $\mathrm{CLA}<\mathrm{CLP} * *$ & $\mathrm{CLA}<\mathrm{CLP} * *$ \\
\hline m1-m1'width & $\mathrm{CLA}<\mathrm{CLP} * *$ & NS \\
\hline \multicolumn{3}{|l|}{ Length of palate (mm) } \\
\hline length & NS & NS \\
\hline \multicolumn{3}{|l|}{ Height of alveolar (mm) } \\
\hline 1height & $\mathrm{CLA}<\mathrm{CLP} *$ & NS \\
\hline 1'height & NS & NS \\
\hline 2height & $\mathrm{CLA}<\mathrm{CLP} *$ & NS \\
\hline 2'height & NS & NS \\
\hline 3height & $\mathrm{CLA}<\mathrm{CLP} *$ & $\mathrm{CLA}<\mathrm{CLP} * *$ \\
\hline 3'height & NS & NS \\
\hline \multicolumn{3}{|l|}{ Height of palate (mm) } \\
\hline 3-3'height & NS & NS \\
\hline 4-4'height & NS & NS \\
\hline 5-5'height & NS & NS \\
\hline 6-6'height & NS & NS \\
\hline \multicolumn{3}{|c|}{ Palate surface area $\left(\mathrm{mm}^{3}\right)$} \\
\hline Map & NS & NS \\
\hline \multicolumn{3}{|c|}{ Palate cross-sectional area $\left(\mathrm{mm}^{3}\right)$} \\
\hline 3-3'sps & NS & NS \\
\hline $4-4$ 'sps & NS & NS \\
\hline $5-5$ 'sps & NS & NS \\
\hline 6-6'sps & NS & NS \\
\hline
\end{tabular}

$\mathrm{NS}=$ not significant; $* \mathrm{p}<0.05 ; * * \mathrm{p}<0.01$ (Mann-Whitney $U$ test)

(1) Intragroup comparison (Tables 1 and 2)

No significant differences were noted at any time in the CLP or CLA group.

(2) Intergroup comparison (Tables 3 and 4)

The height of 5-5' was significantly lower in the CLP group at T1, T2, and T3. No significant differences in the amount of changes were noted between during and at after treatment.

\section{Palatal surface area}

1) Intragroup comparison (Tables 1 and 2)

No significant differences were noted at any of the time points in the CLP or CLA group.

2) Intergroup comparison (Tables 3 and 4) 
Table 5 Correlation coefficients of different changes associated with treatment and retention.

\begin{tabular}{|c|c|c|c|c|}
\hline \multirow{2}{*}{ Parameter } & \multicolumn{2}{|c|}{ Group CLP } & \multicolumn{2}{|c|}{ Group CLA } \\
\hline & r & p-value & $\mathrm{r}$ & p-value \\
\hline \multicolumn{5}{|c|}{ Alveolar arch width (mm) } \\
\hline 3-3'width & -0.429 & NS & -0.310 & NS \\
\hline 4-4'width & 0.973 & $* *$ & 0.929 & $* *$ \\
\hline 5-5'width & -0.905 & $* *$ & -0.619 & NS \\
\hline 6-6'width & 0.190 & NS & -0.500 & NS \\
\hline \multicolumn{5}{|l|}{ Dental arch width $(\mathrm{mm})$} \\
\hline c-c'width & -0.357 & NS & 0.167 & NS \\
\hline p1-p1'width & -0.649 & NS & -0.476 & NS \\
\hline p2-p2'width & -0.81 & $*$ & -0.333 & NS \\
\hline m1-m1'width & -0.738 & $*$ & -0.81 & $*$ \\
\hline \multicolumn{5}{|l|}{ Length of palate (mm) } \\
\hline length & 0.238 & NS & -0.548 & NS \\
\hline \multicolumn{5}{|l|}{ Height of alveolar (mm) } \\
\hline 1height & -0.905 & $* *$ & -0.024 & NS \\
\hline 1'height & -0.119 & NS & 0.095 & NS \\
\hline 2height & -0.852 & $*$ & 0.100 & NS \\
\hline 2'height & -0.200 & NS & 0.600 & NS \\
\hline 3height & -0.929 & $* *$ & 0.262 & NS \\
\hline 3'height & -0.214 & NS & -0.643 & NS \\
\hline \multicolumn{5}{|l|}{ Height of palate $(\mathrm{mm})$} \\
\hline 3-3'height & -0.167 & NS & -0.667 & NS \\
\hline 4-4'height & 0.405 & NS & -0.476 & NS \\
\hline 5-5'height & -0.310 & NS & -0.286 & NS \\
\hline 6-6'height & 0.357 & NS & 0.333 & NS \\
\hline \multicolumn{5}{|c|}{ Palate surface area $\left(\mathrm{mm}^{3}\right)$} \\
\hline Map & -0.167 & NS & -0.452 & NS \\
\hline \multicolumn{5}{|c|}{ Palate cross-sectional area $\left(\mathrm{mm}^{3}\right)$} \\
\hline 3-3'sps & -0.542 & NS & -0.119 & NS \\
\hline 4-4'sps & -0.175 & NS & -0.262 & NS \\
\hline $5-5$ 'sps & -0.452 & NS & -0.714 & * \\
\hline 6-6'sps & 0.238 & NS & 0.143 & NS \\
\hline
\end{tabular}

$\mathrm{NS}=$ not significant; $* \mathrm{p}<0.05 ; * * \mathrm{p}<0.01$ (Spearman's correlation coefficient)

Palatal surface area was significantly smaller at $\mathrm{T} 1$ in the CLP group. No significant differences in the amount of change were noted between during and at after treatment.

\section{Palatal cross-sectional area}

1) Intragroup comparison (Tables 1 and 2)
During the treatment period, 3 sps, 4 sps, 5 sps, and 6 sps showed a significant increase in the CLP group, whereas 5 sps showed a significant decrease during the post-treatment period. In the CLA group, a significant increase was noted in 3 sps during the treatment period. 
2) Intergroup comparison (Tables 3 and 4)

In the CLP group, 4 sps, 5 sps, and 6 sps were significantly lower at $\mathrm{T} 1$, while 5 sps and 6 sps were significantly lower at both T2 and T3 than in the CLA group. No significant differences were noted in the extent of change between during and at post treatment.

\section{Correlations between treatment and post-treatment change (Table 5)}

In the CLP group, the treatment and posttreatment changes showed a correlation at the sites where the 4-4', 5-5', p1-p2', and m1-m1'widths, and the 1,2 , and 3 heights were measured. In the CLA group, the treatment and post-treatment changes showed a correlation at the 4-4' width, m1-m1' width, and 5 sps sites.

\section{Discussion}

\section{Study methods}

\section{1) Patients}

Many researchers have reported that maxillary dental arch patterns in cleft lip and palate patients vary greatly depending on the size of the alveolar cleft, palate closure timing, degree of surgical invasion, and growth potential of each individual patient ${ }^{15,22}$. Such variations could also affect orthodontic treatment, indicating the importance of carrying out a detailed evaluation of the size of the cleft in each patient in selecting the surgical procedure to be applied. It is extremely difficult to obtain such information in Japan, however, due to the absence of a cleft palate center where such patients can be followed up from birth to adulthood. Therefore, it was not possible to ascertain the alveolar cleft size and surgical procedures used in each patient in detail in the present study. There is a lack of consensus regarding the type of surgical procedure to be used in each case and the time at which it should be applied in Japan. To establish a treatment system for patients with cleft lip and cleft palate requiring orthodontic treatment, it is necessary to understand their current situation. The patients included in this study may be considered as a representative sample of the general population of patients seeking orthodontic treatment in Japan. Furthermore, cleft palate surgery can involve either one- or two-stage palatoplasty. In this study, the one-stage method was selected because of the number of patients enrolled. In future research, it will be necessary to compare differences between the oneand two-stage methods.

2) Growth and investigation timing

Maxillary alveolar growth is an important factor in the evaluation of the maxillary arch. Few patients, however, do not undergo orthodontic treatment until growth is completed. Therefore, it was decided to minimize the influence of growth by approximating the age of the patients in the CLP and CLA groups.

3) Retainer

The type and usage time of the retainer are important factors that affect the results of a study. Lang et al. suggested a retention period of at least 2 years for cleft lip and palate patients, and also emphasized the importance of retainers for minimizing relapse ${ }^{8)}$. Therefore, in the present study, specific criteria were established for the selection of patients, all of whom used a circumferential retainer for at least 2 years after debonding and attended regular examinations. Accordingly, it is believed that the results of this study were not influenced by retainer type or usage time. 4) Three-dimensional measurement system

Traditionally, methods of morphological analysis of cleft lip and palate patients have included direct measurement, contour mapping, and cephalometric analysis. However, these conventional methods do not sufficiently employ the 3D data offered by dental casts. Recently, attempts have been made to use a new method of measurement involving a 3D measurement system and a digitizer, with a particular focus on laser digitizers. This type of measurement method offers a number of advantages, including the ability to perform noncontact measurement; the ability to easily measure distance and quantify area using CAD software; and the achievement of high precision measurements. Thus, this system 
appears to be useful in measuring complex palatal morphologies in cleft lip and palate patients.

The strength of this study was the use of 3D elements in calculating anterior alveolar height, palatal height, and palatal cross-sectional area.

5) Measurement points and reference plane

The establishment of a reference plane is important in standardizing the measurement method. In patients with cleft lip and palate, it is difficult to standardize dental measurements because of the large number of deviated and inclined teeth in the oral cavity ${ }^{11)}$. Hence, the method described by Primožič et $a l$. was used in the present $s\left(u d y^{16)}\right.$, which made it easy to establish the reference plane without being influenced by the position of the teeth. The central incisor incisive papilla was established as the anterior reference point, and the deepest points on the palatal sides of the cervices of the left and right first molars established as the posterior reference points.

\section{Results of 3D morphological analysis}

1) Alveolar arch width, dental arch width, and palatal height

The long-term stability of the maxillary dental arch width in cleft lip and palate patients has been investigated previously ${ }^{6,9,17)}$. Scar tissue following surgical closure of the palate is believed to be the most important factor in disturbance of craniofacial growth and development in such patients ${ }^{20,24)}$. In addition, maxillary retrusion with both anterior and lateral crossbite is a very common finding in these patients, regardless of the treatment approach $^{2)}$. Relapse has been reported to be affected by lip pressure and scar tissue, and often occurs in the canine and premolar regions $^{3,6)}$.

In the current study, both alveolar and dental arch widths between the second premolars in the CLP group showed significant relapse; and these sites were significantly smaller than in the CLA group before orthodontic treatment. In addition, the amount of expansion following orthodontic treatment in the CLP group was significantly greater than that in the CLA group. Furthermore, a correlation between the degrees of expansion and relapse was noted, indicating that excessive expansion may have caused the relapse.

The widths of the alveolar and dental arches in the first premolar region were smaller in the CLP group than in the CLA group before orthodontic treatment; the amount of expansion following orthodontic treatment was not significantly different from that in the CLA group, however. Therefore, expansion was not considered to be excessive.

No differences were noted in the first molar regions between the groups before orthodontic treatment. Although the amount of expansion following orthodontic treatment was significantly greater in the CLP group than in the CLA group, no relapse occurred. This might be because excessive expansion in the area of developmental failure causes relapse.

Similar to the results of the study by Athanasios et al., no significant difference in palatal length was noted in the present study ${ }^{27)}$.

2) Height of palate, cross-sectional area, and anterior alveolar area

It has been reported that the palate in cleft lip and palate patients is shallower than in healthy individuals ${ }^{4,14)}$. In the current study, the second premolar area was significantly shallower in the CLP group than in the CLA group. Thus, although many studies have conducted intergroup comparisons in a crosssectional manner, to our knowledge, none to date have involved longitudinal investigations before treatment, during debonding, and at 2 years after debonding. The results of the present study revealed no change in palatal height after orthodontic treatment.

Changes in cross-sectional area were similar to those in alveolar arch width. It has been reported that the anterior maxillary portion near the alveolar cleft is often located at a vertically lower position due to poor growth of the alveolar bone in the maxilla, resulting in lingual or upward relapse during the retention period ${ }^{5,7)}$. There is a dearth of longitudinal studies investigating vertical quantitative 
change in the anterior teeth, however. In the present study, vertical relapse during the retention period was evaluated by measuring the lowest point on the palatal side of the cervical line. In the anterior region, the cleft sides of the central incisor, lateral incisor, and canine in the CLP group showed significant relapse. These sites were significantly higher in the CLP group than in the CLA group before orthodontic treatment. In addition, the amount of extrusion following orthodontic treatment in the CLP group was significantly larger than that in the CLA group. Moreover, a correlation between the amounts of extrusion and relapse was noted. The difference between the alveolar portions in the CLP and CLA groups during the initial period may have been influenced by secondary bone grafting. Generally, the objectives of secondary bone grafting include recovery of alveolar morphology in the alveolar cleft, bone bridge acquisition, maxillary bone continuity and stability, induction of eruption of teeth near the alveolar cleft, and movement of the tooth to the bone graft site ${ }^{10,23)}$. In one study, computed tomography revealed significantly higher amounts of transplanted bone absorption after secondary bone grafting in the CLP group than in the CLA group ${ }^{26)}$.

In the present study, no 3D evaluation of the transplanted bone bridge was performed based on computed tomography. Hence, further studies are required to investigate the correlations between the height and width of the alveolar bone and tooth relapse in the alveolar cleft region.

\section{Conclusion}

A 3D morphological analysis of the maxilla in cleft lip and palate patients who had undergone orthodontic treatment was conducted. Our investigations on the stability of the maxillary arch following expansion revealed the following:

- Use of a laser digitizer makes it possible to measure 3D change in the maxillary alveolar arch.
- Horizontal relapse in the alveolar and dental arches was noted between the second premolars in the CLP group.

- Vertical relapse on the cleft side of the central incisor, lateral incisor, and canine was noted in the CLP group.

- The sites where relapse occurred were associated with poor growth before orthodontic treatment.

- A correlation was observed between the extents of expansion and relapse.

Taken together, these results suggest that excessive vertical or horizontal tooth movement in areas showing developmental failure should be avoided to increase stability after orthodontic treatment. A treatment plan that does not involve expansion of the maxillary arch should be selected whenever possible. In other words, the present findings suggest that reducing scar tissue as much as possible improves the stability of orthodontic treatment.

\section{References}

1) Aiyesha W (2015) Comparison of intercanine and intermolar width between cleft lip palate and normal class I occlusion group. J Coll Physicians Surg Pakistan 25:811-814.

2) Al-Gunaid T, Asahito T, Yamaki M (2008) Relapse tendency in maxillary arch width in unilateral cleft lip and palate patients with different maxillary arch forms. Cleft PalateCraniofacial J 45:278-283.

3) Athanaasios E, Mohammad M, Kourosh Z (1988) Dental arch dimensions in patients with unilateral cleft lip and palate. Cleft PalateCraniofacial J 25:139-145.

4) Ayub PV, Janson G, Gribel BF, Lara TS, Garib DG (2016) Analysis of the maxillary dental arch after rapid maxillary expansion in patients with unilateral complete cleft lip and palate. Am J Orthod Dentofac Orthop 149: 705-715.

5) Bergland O, Semb G, Abyholm FE (1986) Elimination of the residual alveolar cleft by secondary bone grafting and subsequent orthodontic treatment. Cleft Palate-Craniofacial J 23:175-205.

6) Capelozza Filho L (1996) Isolated consequences of lip and palate surgery: a compara- 
tive study in adult males with complete unilateral cleft lip, alveolus and palate. Cleft PalateCraniofacial J 33:51-56.

7) Filho LC, Normando ADC, Da Silva Filho OG (1996) Isolated influences of lip and palate surgery on facial growth: Comparison of operated and unoperated male adults with UCLP. Cleft Palate-Craniofacial J 33:51-56.

8) Lang G, Alfter G, Göz G LG (2002) Retention and stability taking various treatment parameters into account. J Orofac Orthop 63:26-41.

9) Marcusson A, Paulin G (2004) Changes in occlusion and maxillary dental arch dimensions in adults with treated unilateral complete cleft lip and palate: A follow-up study. Eur J Orthod 26:385-390.

10) Meij V Der, Baart JA, Prahl-Andersen B, Valk J, Kostense PJ, Tuinzing DB (2001) Bone volume after secondary bone grafting in unilateral and bilateral clefts determined by computed tomography scans. Oral Surg Oral Med Oral Pathol Oral Radiol Endod 92:136-141.

11) Nicholson P, Plint D (1989) A long-term study of rapid maxillary expansion and bone grafting in cleft lip and palate patients. Eur J Orthod 11:186-192.

12) Noguchi M, Suda Y, Ito S, Kohama G (2003) Dento-alveolar development in unilateral cleft lip, alveolus and palate. J Cranio-Maxillofacial Surg 31:137-141.

13) Normando ADC, da Silva Filho OG, Capelozza Filho L (1992) Influence of surgery on maxillary growth in cleft lip and/or palate patients. J Cranio-Maxillofacial Surg 20:111-118.

14) Oba T, Miki Y, Okazaki M (2004) A study of stability of the maxillary dental arch form with secondary bone grafting in unilateral cleft lip and palete patients. JJpn Cleft Palate Assoc 29: 16-29.

15) Peltomäki T, Vendittelli BL, Grayson BH, Cutting CB, Brecht LE (2001) Associations between severity of clefting and maxillary growth in patients with unilateral cleft lip and palate treated with infant orthopedics. Cleft Palate-Craniofacial J 38:582-586.

16) Primožič J, Perinetti G, Richmond S, Ovsenik M (2012) Three-dimensional longitudinal evaluation of palatal vault changes in growing subjects. Angle Orthod 82:632-636.

17) Ramstad T, Jendal T (1997) A long-term study of transverse stability of maxillary teeth in patients with unilateral complete cleft lip and palate. J Oral Rehabil 24:658-665.

18) Ross RB (1987) Treatment variables affecting facial growth in complete unilateral cleft lip and palate. Part 1: treatment affecting growth. Cleft Palate-Craniofacial J 24:5-23.

19) Rossouw PE, Preston CB, Lombard CJ, Truter JW (1993) A longitudinal evaluation of the anterior border of the dentition. Am J Orthod Dentofac Orthop 104:146-152.

20) Shaw W, Asher-McDade C (1992) A six-center international study of treatment outcome in patients with clefts of the lip and palate: Part 1 . Principles and study design. Cleft PalateCraniofacial J 29:405-408.

21) Susami T (2006) Assessment of dental arch relationships in Japanese patients with unilateral cleft lip and palate. Cleft PalateCraniofacial J 43:96-102.

22) Suzuki A, Mukai Y, Ohishi M, Miyanoshita Y, Tashiro H (1993) Relationship between cleft severity and dentofacial morphology in Japanese subjects with isolated cleft palate and complete unilateral cleft lip and palate. Cleft Palate-Craniofacial J 30:178-181.

23) Toscano D, Baciliero U, Gracco A, Siciliani G (2012) Long-term stability of alveolar bone grafts in cleft palate patients. Am J Orthod Dentofac Orthop 142:289-299.

24) Trotman CA, Collett AR, McNamara Jr. JA, Cohen SR (1993) Analyses of craniofacial and dental morphology in monozygotic twins discordant for cleft lip and unilateral cleft lip and palate. Angle Orthod 63:135-139.

25) Uhde MD, Sadowsky C, Begole EA (1983) Long-term stability of dental relationships after orthodontic treatment. Angle Orthod 53:240-252.

26) Van Der Meij AJW, Baart JA, Prahl-Andersen B, Valk J, Kostense PJ, Tuinzing DB (2001) Bone volume after secondary bone grafting in unilateral and bilateral clefts determined by computed tomography scans. Oral Med Oral Pathol Oral Radiol Endod 92:136-141.

27) Zbynek S (2004) Three-dimensional morphology of the palate in subjects with unilateral complete cleft lip and palate at the stage of permanent dentition. Cleft Palate-Craniofacial J 41:416-423.

Correspondence:

Dr. Issei Takahashi

Department of Orthodontics,

Tokyo Dental College,

2-9-18 Kanda-Misakicho, Chiyoda-ku,

Tokyo 101-0061, Japan

E-mail: takahashiisseiortho@gmail.com 